\title{
Article
}

\section{Co-precipitation synthesis of reduced graphene oxide/NiAl-layered double hydroxide hybrid and its application in flame retarding poly(methyl methacrylate)}

Hong, Ningning, Song, Lei, Wang, Bibo, Stec, Anna A, Hull, T Richard, Zhan, Jing and Hu, Yuan

Available at http://clok.uclan.ac.uk/10865/

Hong, Ningning, Song, Lei, Wang, Bibo, Stec, Anna A ORCID: 0000-0002-68610468, Hull, T Richard ORCID: 0000-0002-7970-4208, Zhan, Jing and Hu, Yuan (2014) Co-precipitation synthesis of reduced graphene oxide/NiAl-layered double hydroxide hybrid and its application in flame retarding poly(methyl methacrylate). Materials Research Bulletin, 49 (1). pp. 657-664. ISSN 00255408

It is advisable to refer to the publisher's version if you intend to cite from the work. http://dx.doi.org/10.1016/j.materresbull.2013.09.051

For more information about UCLan's research in this area go to http://www.uclan.ac.uk/researchgroups/ and search for <name of research Group>.

For information about Research generally at UCLan please go to http://www.uclan.ac.uk/research/

All outputs in CLoK are protected by Intellectual Property Rights law, including Copyright law. Copyright, IPR and Moral Rights for the works on this site are retained by the individual authors and/or other copyright owners. Terms and conditions for use of this material are defined in the policies page. 
Ningning Hong, Lei Song, Bibo Wang, Anna A. Stec, T. Richard Hull, Jing Zhan, Yuan Hu,

Co-precipitation synthesis of reduced graphene oxide/NiAl-layered double hydroxide hybrid and its application in flame retarding poly(methyl methacrylate), Materials Research Bulletin, 49, 2014, Pp 657-664, http://dx.doi.org/10.1016/j.materresbull.2013.09.051

\title{
Co-precipitation synthesis of reduced graphene oxide/NiAl-layered double hydroxide hybrid and its application in flame retarding poly(methyl methacrylate)
}

\author{
Ningning Hong ${ }^{a}$, Lei Song ${ }^{a}$, Bibo Wang ${ }^{a}$, Anna A. Stec ${ }^{b}$, T. Richard Hull ${ }^{b}$, Jing Zhan ${ }^{a}$, Yuan Hu \\ a State Key Laboratory of Fire Science, University of Science and Technology of China, Hefei, Anhui 230026, PR China \\ ${ }^{\mathrm{b}}$ Centre for Fire and Hazards Science, University of Central Lancashire, Preston PR1 2HE, UK
}

\begin{abstract}
A reduced graphene oxide/NiAl-layered double hydroxide (RGO-LDH) was synthesized through a simple co-precipitation route. NiAl-layered double hydroxide (NiAl-LDH) nanoparticles were homogeneously dispersed on the reduced graphene oxide (RGO) nanosheets, which were simultaneously reduced during the process. RGO-LDH exhibited three steps of weight loss, leaving high residue. RGO-LDH was then solution blended into poly(methyl methacrylate) (PMMA) to investigate its effect on reducing flammability of the composite. With the incorporation of RGO-LDH, the thermal stability of PMMA composite was improved. Moreover, RGO-LDH endowed PMMA with the largest reduction in the heat release rate, smoke production and $\mathrm{CO}$ production rate relative to RGO or NiAl-LDH alone. RGO-LDH could decrease the production of volatiles including hydrocarbons, carbonyl compounds and epoxy compounds from the PMMA composite. The improved flame retardancy was ascribed to the combined effect of the physical barrier of RGO and the catalytic carbonization of NiAl-LDH.
\end{abstract}

\section{Introduction}

As an emerging two-dimensional material, graphene, consisting of monolayer of $\mathrm{sp}^{2}$-hybridized carbon atoms, has currently been the focus of research due to its unique mechanical, thermal and electrical properties [1]. One important application is the use of graphene to reduce the flammability of polymers. It has been reported that addition of graphene confers good flame retardancy to certain polymers similar to other typical nanofillers, such as carbon nanotubes and montmorillonite [2] and [3]. Such improvement might be attributed to the physical barrier effect of layered graphene which slows down the release of flammable gases and protects the underlying matrix from further burning. Huang's group has reported the use of graphene as flame retardant additive in polymers [2] and [4]. Wu et al. found that the pyrolysis temperature of polystyrene was significantly increased with the incorporation of reduced graphene oxide (RGO) [5]. Thermal RGO onto which polypropylene has been grafted was not only easily dispersed in polymers, but also dramatically improved the thermal stability [6]. However, there are at least two problems hindering the application of graphene as a flame retardant: (1) although graphene shows a promising prospect as a flame retardant, its efficiency is not enough to meet many requirements when graphene is used alone [7]; (2) the restacking of as-reduced graphene, due to van der Waals forces, limits the homogeneous dispersion of graphene in the matrix [8] and [9].

Another class of lamellar materials, layered double hydroxide (LDH), has received much attention in recent years due to its special hydrotalcite-like structure. The basic building unit of LDH is brucite 
layers. The partial replacement of interlayer metal ions by trivalent ones leads to an excess positive charge [10] and [11]. Therefore, hierarchical nanostructures based on LDH and other nanomaterials are usually assembled through electrostatic interaction [12]. One big advantage of LDH is the highly tunable properties realized by changing the interlayer composition and ratio of the metals, which makes its use possible in catalysis, pollutant treatment, flame retardancy and medical materials [10] and [13]. The effect of chemical composition and the dispersion of LDH on the flame retardancy of thermoplastic polymers, especially poly(methyl methacrylate) (PMMA), was investigated in detail. Previous report demonstrated that LDH containing transitional metals could be well dispersed in PMMA, and also enhanced thermal stability and reduced flammability could be achieved [14]. However, LDH reinforced PMMA composites still remain a challenge to flame retard efficiently.

Fang et al. have investigated the synergistic effect between carbon nanotubes and LDH on the flame retardancy of polypropylene and observed a noticeable reduction in the peak heat release rate (PHRR) [15]. There were already some reports about the fabrication of graphene/LDH nanocomposites with enhanced electrochemical performance and adsorption capacity [16] and [17]. Inspired by the synergistic effect between LDH and carbon nanomaterials, we have synthesized a hybrid of RGO and LDH containing a transitional metal, for example RGO/NiAl-LDH, in an attempt to design a more efficient flame retardant. In addition, we believe that the distribution of LDH on the surface of RGO could hinder the restacking of interlayer nanosheets, thus increasing the degree of an exfoliation [18].

It is well known that the RGO has a pronounced tendency to agglomerate after drying owing to its high surface energy. Fortunately, the remaining functional groups make RGO hydrophobic, which allows it to disperse readily in organic solvents. Also, the oxygen functionality increases the compatibility between RGO and certain polar polymers. Therefore, solution mixing has been widely adopted for preparation of graphene filled PMMA, polyurethane and polyamide composites with significant enhancement [19], [20] and [21]. In addition, the presence of polar groups on the LDH structure is also beneficial for the good dispersion in PMMA matrix, thus the dispersion state of the hybrid will be greatly improved.

In this study, a one-pot co-precipitation method was used to fabricate a hybrid consisted of RGO and NiAl-LDH (RGO-LDH) and its structure was characterized in detail. The RGO-LDH hybrid was then incorporated into PMMA to investigate the combined effect of RGO and NiAl-LDH on the flame retardancy of the composite. It was anticipated that the hybrid could impart PMMA with better flame retardancy due to the physical barrier of RGO and the catalytic carbonization of NiAl-LDH.

\section{Experimental}

\subsection{Materials}

Natural graphite powder (Spectrum Pure), $\mathrm{Ni}\left(\mathrm{NO}_{3}\right)_{2} \cdot 6 \mathrm{H}_{2} \mathrm{O}, \mathrm{Al}\left(\mathrm{NO}_{3}\right)_{3} \cdot 9 \mathrm{H}_{2} \mathrm{O}, \mathrm{Na}_{2} \mathrm{CO}_{3}, \mathrm{NaOH}$, and $\mathrm{CHCl}_{3}$ were purchased from Sinopharm Chemical Reagent Co., Ltd. (China). $\mathrm{KMnO}_{4}, \mathrm{NaNO}_{3}$, $\mathrm{H}_{2} \mathrm{SO}_{4}(98 \%), \mathrm{N}_{2} \mathrm{H}_{4} \cdot \mathrm{H}_{2} \mathrm{O}, \mathrm{H}_{2} \mathrm{O}_{2}(30 \%)$ and $\mathrm{HCl}(37 \%)$ were bought from Guangfu Fine Chemial Research Institute (Tianjin, China). PMMA was provided by Shenma Company (Henan, China).

\subsection{Preparation of RGO-LDH}

GO was prepared from oxidation of graphite powder by Hummers's method [22]. The RGO-LDH hybrid was prepared by a one-pot co-precipitation process. Briefly, $\mathrm{GO}(0.32 \mathrm{~g})$ was dispersed into a flask containing $150 \mathrm{~mL}$ of $\mathrm{NaOH}(0.20 \mathrm{M})$ and $\mathrm{Na}_{2} \mathrm{CO}_{3}(0.05 \mathrm{M})$ solution by ultrasonication. Then, $\mathrm{Ni}\left(\mathrm{NO}_{3}\right)_{2} \cdot 6 \mathrm{H}_{2} \mathrm{O}(0.075 \mathrm{M})$ and $\mathrm{Al}\left(\mathrm{NO}_{3}\right)_{3} \cdot 9 \mathrm{H}_{2} \mathrm{O}(0.025 \mathrm{M})$ dispersed in $150 \mathrm{~mL} \mathrm{H} \mathrm{H}_{2} \mathrm{O}$ was added into the above mixture with vigorous stirring. Subsequently, the $\mathrm{pH}$ value of the solution was adjusted to 10.5 by addition of $0.2 \mathrm{M} \mathrm{NaOH}$ solution. The mixture was first heated to $60^{\circ} \mathrm{C}$ and then stirred at reflux temperature $\left(95^{\circ} \mathrm{C}\right) \cdot 0.75 \mathrm{~mL}$ of $\mathrm{N}_{2} \mathrm{H}_{4} \cdot \mathrm{H}_{2} \mathrm{O}$ was then added quickly to the above solution. After refluxing for $6 \mathrm{~h}$, the suspension was centrifuged, washed, and dried at $60^{\circ} \mathrm{C}$. As a comparison, NiAlLDH and RGO were prepared under the similar experimental condition. 


\subsection{Preparation of PMMA composites}

The PMMA based composites were prepared by a solution-blending method. Typically, $0.8 \mathrm{~g}$ of RGO$\mathrm{LDH}$ was dispersed in $\mathrm{CHCl}_{3}$ by bath sonication to form a uniform suspension. Afterwards, $39.2 \mathrm{~g}$ of PMMA pellets were added to the suspension and mechanically stirred at $80^{\circ} \mathrm{C}$ for $3 \mathrm{~h}$. Then, the PMMA/RGO-LDH was cast into Petri dish and dried at $60{ }^{\circ} \mathrm{C}$ for $12 \mathrm{~h}$ to remove the solvent. Other samples containing $0.4 \mathrm{wt} . \%$ graphene and $2 \mathrm{wt} . \% \mathrm{NiAl}-\mathrm{LDH}$ were prepared using the same procedure. Finally, the samples were hot-pressed at $200{ }^{\circ} \mathrm{C}$ and $10 \mathrm{MPa}$ to form sheets with suitable sizes.

\subsection{Measurements and characterization}

X-ray diffraction (XRD) patterns were obtained using a Japan Rigaku D/Max-Ra rotating anode X-ray diffractometer equipped with a Cu-Ka tube and Ni filter $(\lambda=0.1542 \mathrm{~nm})$.

Fourier transform infrared (FTIR) spectra of the samples were recorded on a Nicolet 6700 spectrometer (Nicolet Instrument Company) in the $500-4000 \mathrm{~cm}^{-1}$ region.

Raman spectroscopy was carried out with a SPEX-1403 laser Raman spectrometer (SPEX Co., USA) with excitation provided in back-scattering geometry by a $514.5 \mathrm{~nm}$ argon laser line.

Transmission electron microscopy (TEM) micrographs and selected area electron diffraction (SAED) were obtained by JEOL 2010 with an acceleration voltage of $200 \mathrm{kV}$. Specimens for the high resolution electron microscopy (HRTEM) measurements were obtained by dripping the suspension onto a Cu grid supported carbon film.

The morphology of the sample after being gold-sputtered was studied by a PHILIPS XL30E scanning electron microscopy. The specimens of PMMA composites were cryogenically fractured at room temperature, and then sputter-coated with the conductive layer.

Thermogravimetric analysis (TGA) was carried out on the TGA Q5000 IR thermogravimetric analyzer (TA instruments) using a heating rate of $20^{\circ} \mathrm{C} / \mathrm{min}$ in nitrogen and/or air.

The combustion test was performed on the cone calorimeter (FTT, UK) test in accordance with ISO 5660 standard. Each specimen was wrapped in an aluminium foil and exposed horizontally to $35 \mathrm{~kW} / \mathrm{m}^{2}$ external heat flux.

Thermogravimetric analysis/infrared spectrometry (TG-IR) of the samples was performed using the TGA Q5000 IR thermogravimetric analyzer that was interfaced to the Nicolet 6700 FTIR spectrophotometer.

\section{Results and discussion}

The crystal structures of the GO, RGO, NiAl-LDH and RGO-LDH hybrid were characterized by XRD (Fig. 1). The XRD pattern of $G O$ exhibits a sharp peak at $10.4^{\circ}$ indicating the interplanar spacing of the $\left(\begin{array}{ll}0 & 0\end{array}\right)$ is about $0.85 \mathrm{~nm}$. After the co-precipitation process, the peak for $\left(\begin{array}{ll}0 & 0\end{array}\right)$ plane is shifted to $24.8^{\circ}$, because the oxygen-containing groups in the structure are removed. The width of the peak is attributed to a turbostratic arrangement of graphene stacked sheets [23]. The diffraction peaks at $11.4^{\circ}, 23.1^{\circ}, 35.2^{\circ}, 39.3^{\circ}$ and $46.5^{\circ}$ are ascribed to the (l 003$),\left(\begin{array}{lll}0 & 0 & 6\end{array}\right),\left(\begin{array}{lll}0 & 1 & 2\end{array}\right),\left(\begin{array}{lll}0 & 1 & 5\end{array}\right)$ and $\left(\begin{array}{lll}0 & 1 & 8\end{array}\right)$ planes of typical hydrotalcite-like NiAl-LDH [24]. The basal spacing around $7.7 \AA$ calculated from the $\left(\begin{array}{lll}0 & 0 & 3\end{array}\right)$ plane indicates the intercalation of $\mathrm{CO}_{3}{ }^{2-}$ ions into the interlayer spaces. The XRD pattern of RGO-LDH hybrid is almost identical to that of pure NiAl-LDH, and no characteristic peak of RGO is observed. These results indicate that the NiAl-LDH is well anchored on the RGO sheets, which effectively prevents the restacking of the as-reduced graphene [18]. 


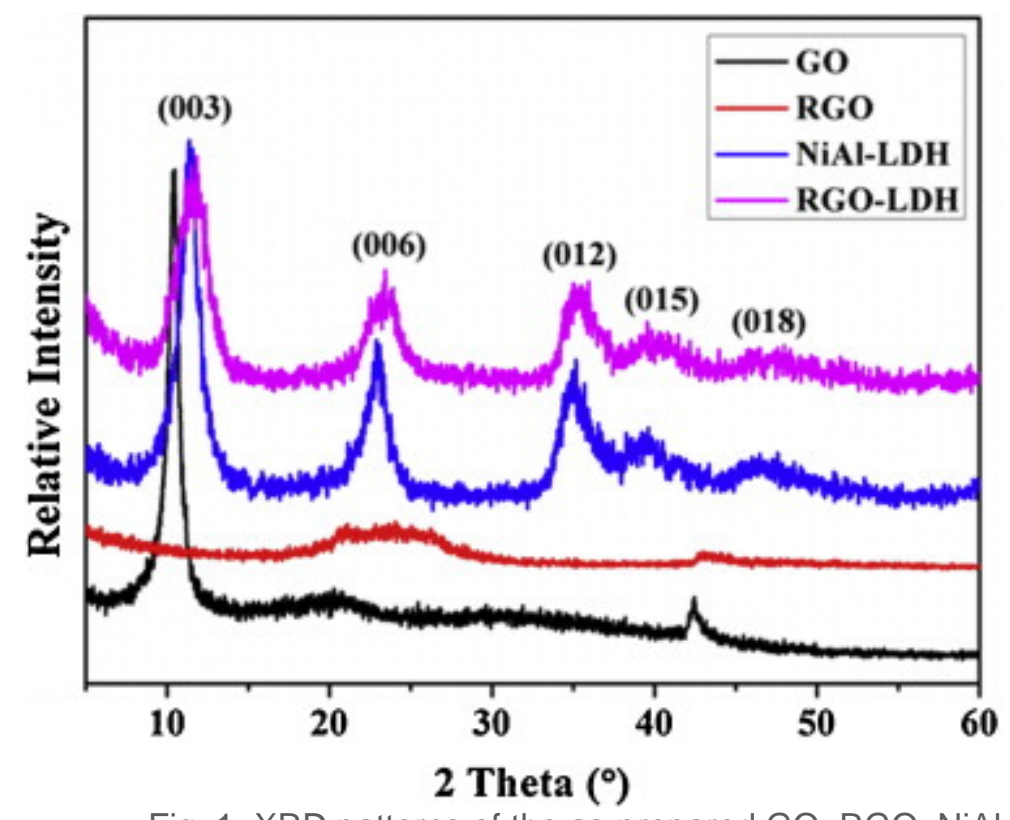

Fig. 1. XRD patterns of the as-prepared GO, RGO, NiAl-LDH and RGO-LDH respectively.

Fig. 2 shows the FTIR spectra of the RGO-LDH hybrid together with GO, RGO and NiAl-LDH. GO shows characteristic peaks at 3416, 1722, 1592, 1226 and $1047 \mathrm{~cm}^{-1}$, which are assigned to the O-H stretch, carbonyl $\mathrm{C}=\mathrm{O}$ stretch, aromatic $\mathrm{C}=\mathrm{C}$ stretch, epoxy $\mathrm{C}-\mathrm{O}$ stretch and alkoxy $\mathrm{C}-\mathrm{O}$ stretch, respectively. After the co-precipitation process, the peaks of the oxygen functional groups almost disappear. The FTIR spectrum of RGO only displays three weak peaks at 3435, 1564 and $1203 \mathrm{~cm}^{-1}$ corresponding to the $\mathrm{O}-\mathrm{H}$ stretch, aromatic $\mathrm{C}=\mathrm{C}$ stretch and $\mathrm{C}-\mathrm{O}$ stretch respectively. For the $\mathrm{NiAl}-\mathrm{LDH}$, the broad bands around 3453 and $1635 \mathrm{~cm}^{-1}$ are attributed to the stretching mode and bending mode of the $-\mathrm{OH}$ groups of the LDH layer, respectively. The bands around 1369 and $762 \mathrm{~cm}^{-1}$ are assigned to the vibration of $\mathrm{CO}_{3}{ }^{2-}\left(\mathrm{v}_{3}\right)$ and $\mathrm{CO}_{3}{ }^{2-}\left(\mathrm{v}_{2}\right)$, indicative of the characteristics of interlayer carbonate anions. Furthermore, the absorption band below $700 \mathrm{~cm}^{-1}$ is ascribed to the characteristic peak of $\mathrm{Ni}$ _ $\mathrm{O}$ and $\mathrm{Al} \_\mathrm{O}$ vibrations in the lattice of $\mathrm{LDH}$ [11] and [25]. As shown in Fig. 2, almost all the characteristic peaks corresponding to NiAl-LDH are observed in the spectrum of RGO-LDH. Compared with NiAl-LDH, there is a shift in the peak location of RGO-LDH alongside the decreased absorption intensities due to the incorporation of RGO.

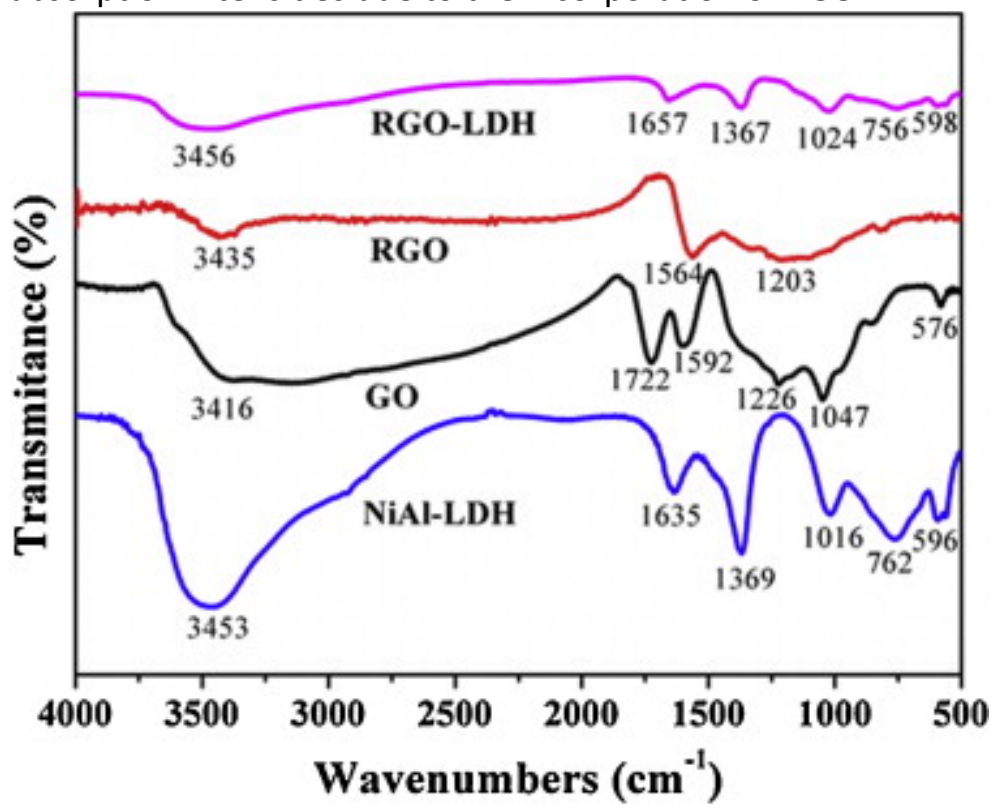

Fig. 2. FTIR spectra of the GO, RGO, NiAl-LDH, and RGO-LDH hybrid. 
Raman spectroscopy was used to investigate the structural changes of the samples during the coprecipitation process. In carbonaceous material, there are two prominent $D$ and $G$ bands corresponding to the defect structures and $\mathrm{sp}^{2}$ carbon, and their intensity ratio $\left(I_{\mathrm{D}} / \mathrm{I}_{\mathrm{G}}\right)$ is a measure of disorder degree and average size of the $\mathrm{sp}^{2}$ domains [26]. Fig. 3 shows the Raman spectra of GO, RGO, NiAl-LDH and RGO-LDH samples. GO displays a strong $D$ band at $1354 \mathrm{~cm}^{-1}$ and a weak $G$ band at $1632 \mathrm{~cm}^{-1}$. A decreased $I_{\mathrm{D}} / I_{\mathrm{G}}$ value (1.35) is observed for RGO, indicative of partial recovering of the graphitic structure. The bands of NiAl-LDH at $555 \mathrm{~cm}^{-1}$ and $1063 \mathrm{~cm}^{-1}$ originate from the hydroxyl layers and stretching mode of $\mathrm{CO}_{3}{ }^{2-}$ of the $\mathrm{LDH}$ [27]. In the case of the RGO-LDH, the $I_{D} / I_{G}$ value (1.86) is increased as compared to $G O$, indicating a decrease in the size of $\mathrm{sp}^{2}$ domains.

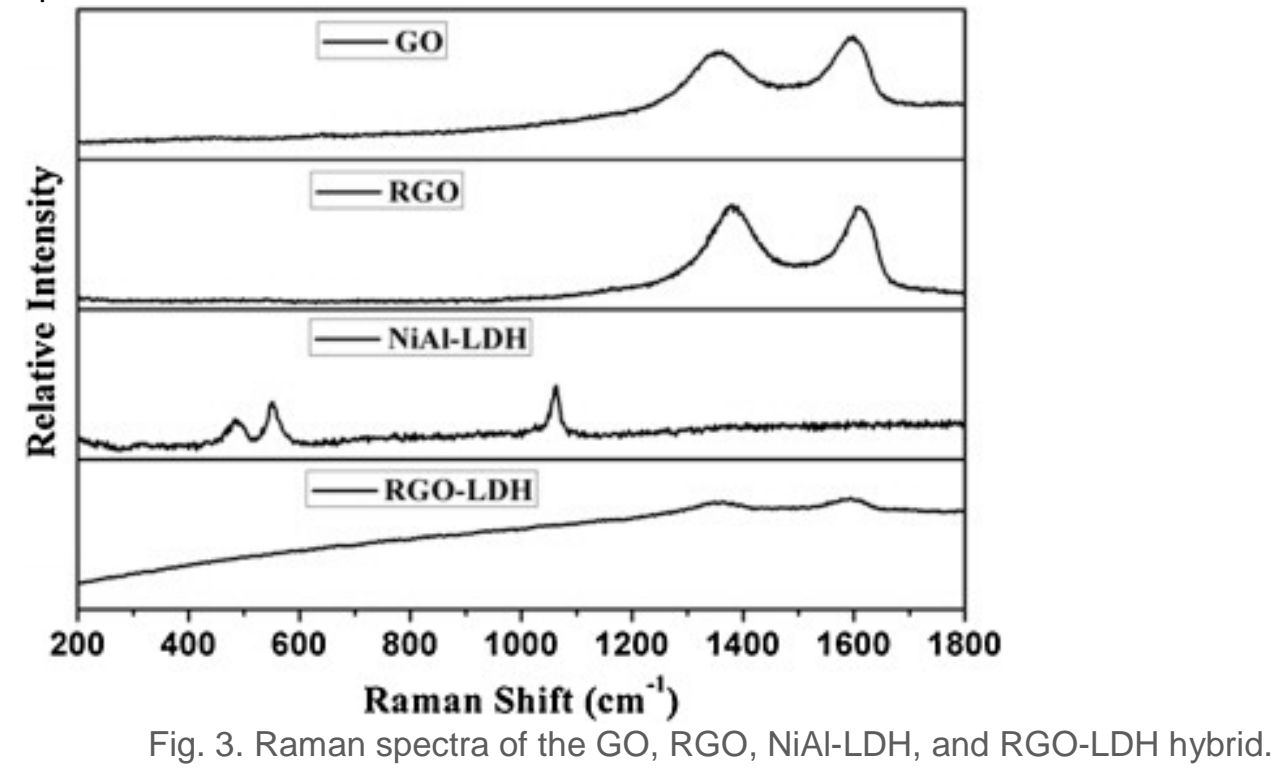

TEM observations were employed to characterize the morphologies of RGO, NiAl-LDH and RGO-LDH hybrid. As shown in Fig. 4a, RGO consists of thin carbon nanosheets with lateral size of several hundred nanometers. Some of the graphene layers are folded over each other. Fig. $4 \mathrm{~b}$ indicates that $\mathrm{NiAl-LDH}$ is plate-like with a microscopically smooth surface. The thin sheets are almost regular hexagons with a mean diameter of about $300 \mathrm{~nm}$. In the case of RGO-LDH hybrid, the surfaces of RGO sheets are homogeneously attached by many nanoparticles (Fig. 4c). The size of nanoparticles is uniformly distributed in the range of $6-10 \mathrm{~nm}$ due to the presence of graphene templates [18]. As can be seen, the restacking of RGO nanosheets is effectively prevented due to the attachment of $\mathrm{NiAl}-\mathrm{LDH}$ particles. SAED shown in the inset of Fig. 4c was performed on RGO-LDH hybrid. The diffraction rings in this region can be ascribed to the layered structure of $L D H$, which is consistent with XRD analysis [28]. HRTEM measurement of RGO-LDH hybrid (Fig. 4d) indicates that the RGO supported LDH nanoparticles are well crystallized. The lattice fringes with a distance of $0.75 \mathrm{~nm}$ are indicated by white lines, which can be indexed as the (1 110$)$ plane of crystallized LDH phase. 

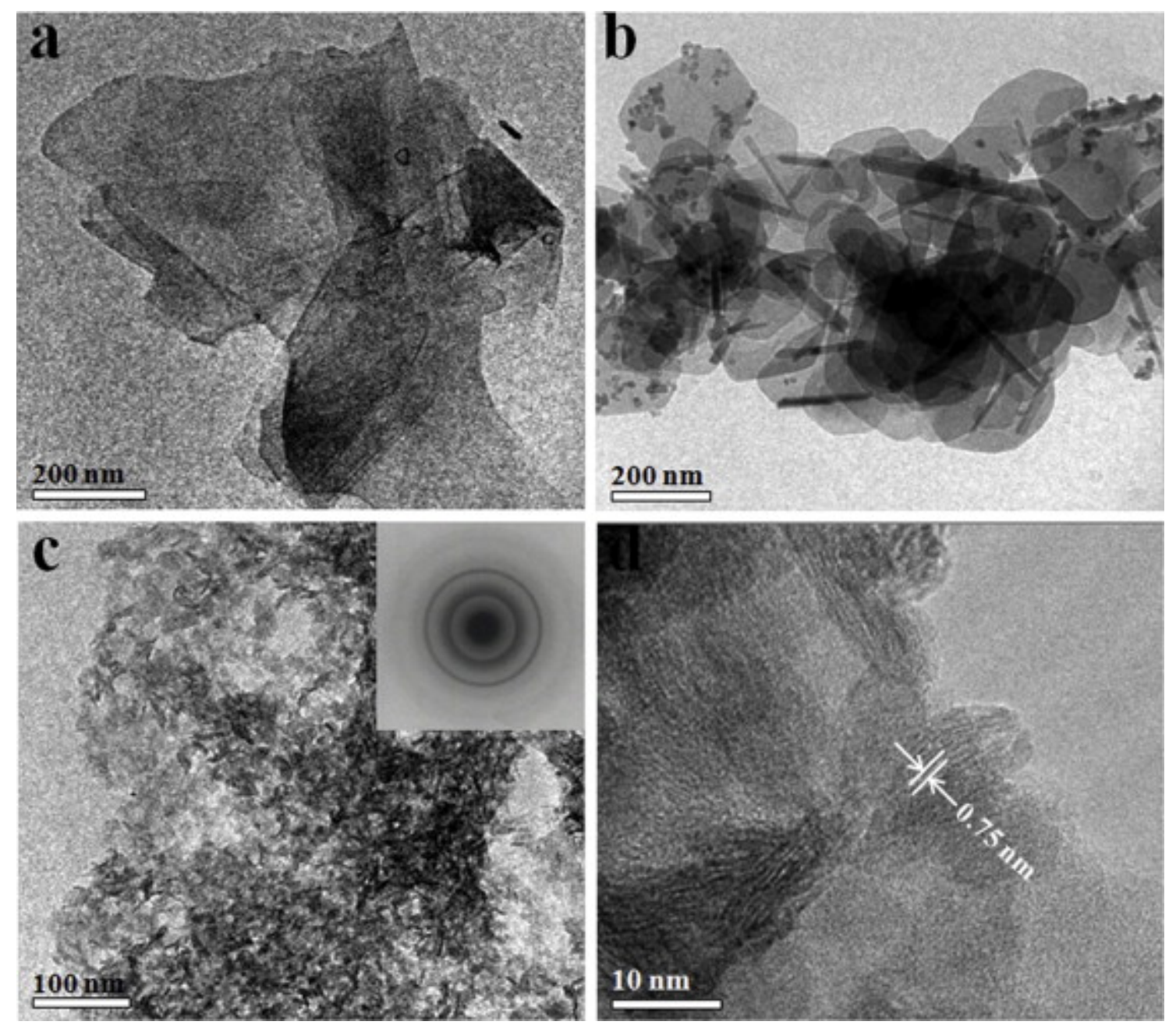

Fig. 4. TEM images of RGO (a), NiAl-LDH (b), RGO-LDH (c) and HRTEM image of RGO-LDH hybrid (d). The inset in panel $c$ is the SAED pattern of RGO-LDH.

Fig. 5 shows the TGA and derivative thermogravimetric (DTG) curves of RGO-LDH in both nitrogen and air. Summary data are listed in Table 1. The temperature of maximum degradation for each step is taken as $T_{\max }$. The RGO-LDH exhibits three distinct steps of weight loss: The first weight loss from 100 to $300{ }^{\circ} \mathrm{C}$ corresponds to the release of water adsorbed and intercalated in the RGO-LDH; the second step takes place in the temperature range from 300 to $500{ }^{\circ} \mathrm{C}$, attributed to the dehydroxylation of the $\mathrm{LDH}$ layers and removal of $\mathrm{CO}_{2}$ from the interlayer carbonate anions [29]; at high temperature $\left(500-700^{\circ} \mathrm{C}\right)$, different reactions occur: the weight loss in nitrogen is ascribed to the reduction of $\mathrm{Ni}^{2+}$ to $\mathrm{Ni}^{0}$ by carbon, while in air it may result from the combustion of the RGO skeleton [30]. 


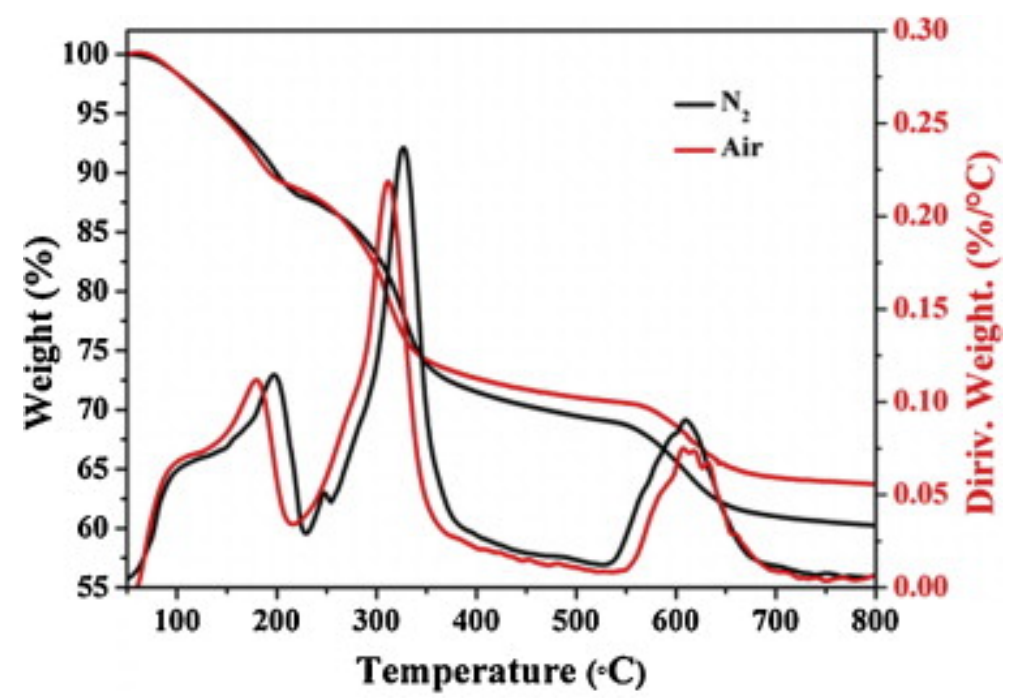

Fig. 5. TGA and DTG curves of RGO-LDH in both nitrogen and air atmosphere.

Table 1. TGA data for the RGO-LDH hybrid in both nitrogen and air atmosphere.

\begin{tabular}{|l|l|l|l|l|}
\hline Atmosphere & $T_{\max 1}\left({ }^{\circ} \mathrm{C}\right)$ & $T_{\max 2}\left({ }^{\circ} \mathrm{C}\right)$ & $T_{\max 3}\left({ }^{\circ} \mathrm{C}\right)$ & Residue at $800{ }^{\circ} \mathrm{C}($ wt.\%) \\
\hline $\mathrm{N}_{2}$ & 197 & 327 & 609 & 60.2 \\
\hline Air & 180 & 312 & 618 & 63.7 \\
\hline
\end{tabular}

The dispersion state and interfacial interaction are considered to be the controlling factors that affect various properties of polymer composites. In order to investigate the dispersion of the hybrid in PMMA, SEM was used to observe the morphology of the composites. Fig. 6 shows the SEM images of the fractured surfaces for pure PMMA and its composites with hybrid. PMMA is an amorphous glassy polymer, thus its fracture surface exhibits frangible features (Fig. 6a). However, some scaly patterns are observed on the surface of PMMA/RGO composite, which implies that the RGO is tightly adhered to the matrix (Fig. 6b) [31]. The strong interaction arises from the hydrogen bonds between the remaining oxygen-containing groups of RGO and the carbonyl groups of PMMA. As revealed in Fig. 6c, some irregular protuberances are observed in the NiAl-LDH reinforced PMMA composite. Similarly, the PMMA/RGO-LDH composite presents a rough fracture surface with narrow bands visible, indicating good compatibility between RGO-LDH and PMMA (Fig. 6d) [32]. This demonstrates that the restacking and aggregation of RGO hybrid is prevented by the applied solution mixing. 

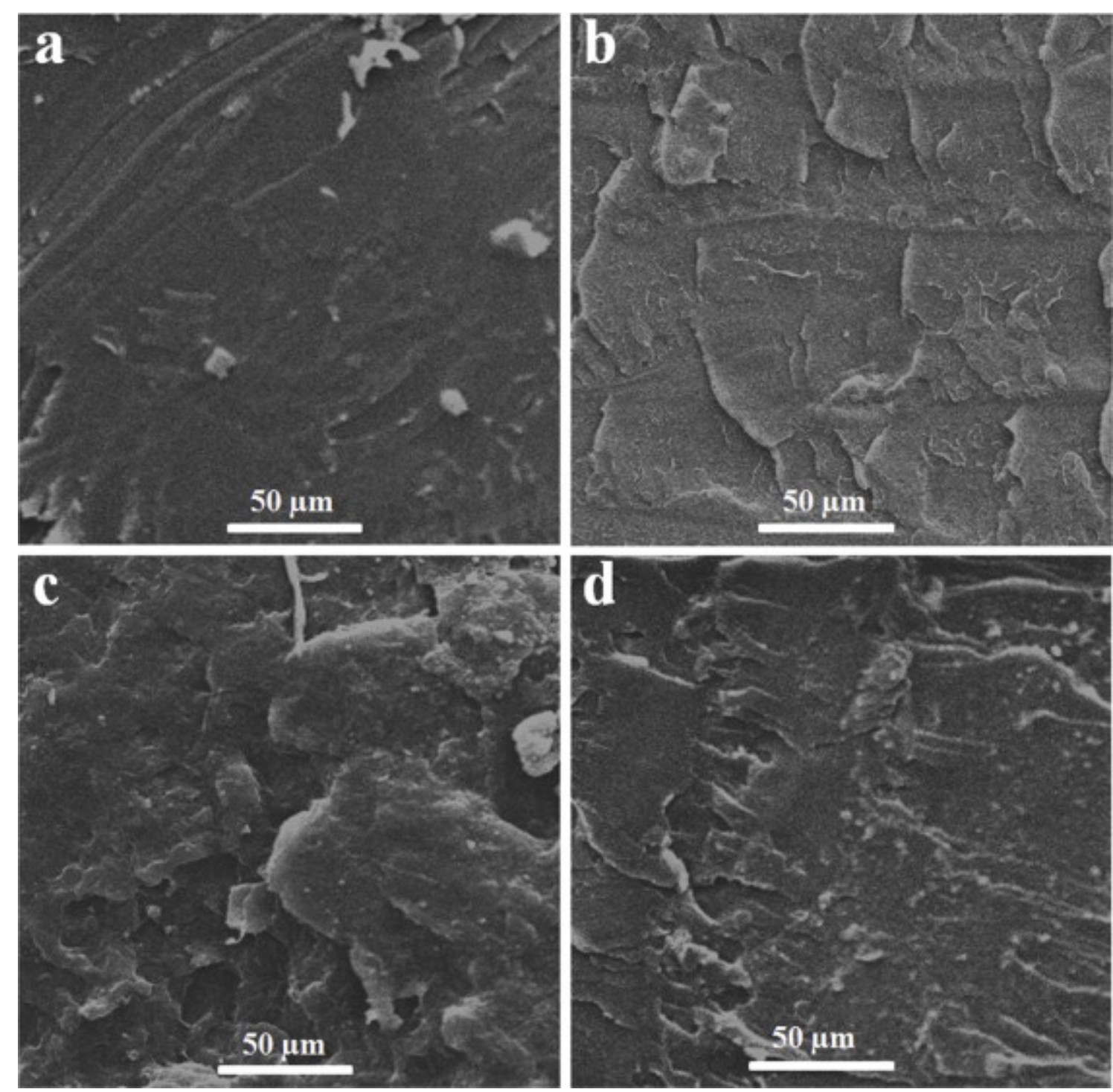

Fig. 6. SEM images for the fractured surfaces of pure PMMA (a), PMMA/RGO composite (b) PMMA/NiAl-LDH composite (c) and PMMA/RGO-LDH composite (d).

The influence of RGO-LDH on the thermal stability of PMMA was investigated by TGA in air atmosphere. Fig. 7 shows the TGA (a) and DTG (b) curves for pure PMMA, PMMA/RGO, PMMA/NiAl$\mathrm{LDH}$ and PMMA/RGO-LDH. The detailed data are listed in Table 2. Thermal degradation of pure PMMA shows only one step and no residue remains. After the addition of RGO, the $T_{-5 \%}$ (defined as the temperature where $5 \%$ of weight loss occurs) decreases by $12{ }^{\circ} \mathrm{C}$ due to the high thermal conductivity of RGO. However, the maximum decomposition rate decreases slightly with little residue remaining perhaps because the RGO inhibits final thermal degradation of PMMA. In the case of PMMA/NiAl-LDH composite, more char residue ( $2.2 \mathrm{wt} . \%)$ is left due to the catalytic carbonization effect of LDH [33]. Enhanced thermal stability of PMMA is obtained as observed by the greater residue of $3.7 \mathrm{wt} . \%$, which is attributed to the combination of the physical barrier effect of RGO and the catalytic carbonization of NiAl-LDH [15]. 

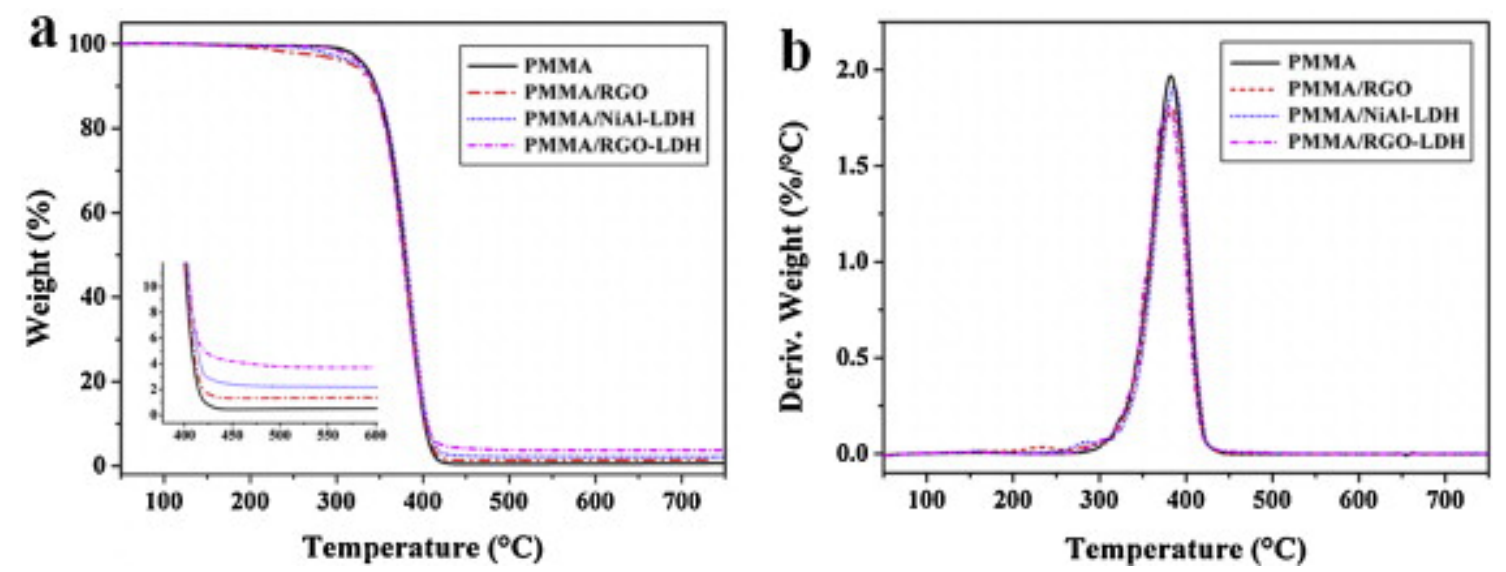

Fig. 7. TGA (a) and DTG (b) curves for pure PMMA, PMMA/RGO composite, PMMA/NiAlLDH composite and PMMA/RGO-LDH composite in air. The inset in (a) shows an enlarged $Y$ axis of weight remaining.

Table 2. TGA data for pure PMMA, PMMA/RGO composite, PMMA/NiAl-LDH composite and PMMA/RGO-LDH composite in air.

\begin{tabular}{|l|l|l|l|}
\hline Sample & $T_{-5 \%}\left({ }^{\circ} \mathrm{C}\right)$ & $T_{\max }\left({ }^{\circ} \mathrm{C}\right)$ & Residue at $750{ }^{\circ} \mathrm{C}$ (wt.\%) \\
\hline PMMA & 332 & 382 & 0.6 \\
\hline PMMA/RGO & 320 & 384 & 1.4 \\
\hline PMMA/NiAI-LDH & 326 & 386 & 2.2 \\
\hline PMMA/RGO-LDH & 330 & 380 & 3.7 \\
\hline
\end{tabular}

The flammability of the composites was characterized by cone calorimetry, which is useful for studying their fire behaviors [34]. Important parameters include the time to ignition (TTI), heat release rate (HRR), CO production rate (COPR), average specific extinction area (ASEA) and peak CO yield (PCOY). Fig. 8 shows the plots of HRR and COPR of PMMA and its composites and their data are summarized in Table 3. Pure PMMA is easily ignited (TTI = $31 \mathrm{~s}$ ) and quickly burns up with a sharp PHRR value of $918 \mathrm{~kW} / \mathrm{m}^{2}$. It is clear that the addition of RGO in the PMMA leads to a $17 \%$ reduction in the PHRR and a 79\% reduction in the PCOY, but shows little effect on TTI and ASEA. Such reduction may be attributed to the barrier effect of RGO in the matrix which slows down the combustion process [2] and [35]. For the PMMA/NiAI-LDH composite, a relatively lower reduction in the PHRR and PCOY is observed, but the TTI is increased to $34 \mathrm{~s}$. The flame retardancy is attributed to the endothermic heat of the decomposition of LDH and the catalytic carbonization effect of the NiAl-oxide formed [36]. It is worth noting that RGO-LDH further reduces the PHRR to $688 \mathrm{~kW} / \mathrm{m}^{2}$ with respect to RGO or NiAl-LDH alone, indicating that the combination of two additives imparts better flame retardancy to PMMA [7]. The TTI of PMMA/RGO-LDH is longer than that of pure PMMA. Furthermore, the values of ASEA and PCOY of PMMA/RGO-LDH composite are lower than those of other composites, which indicates that the loading of LDH onto the surface of RGO improves the catalytic efficiency in reducing fire hazards, such as smoke and toxic gases [37]. 

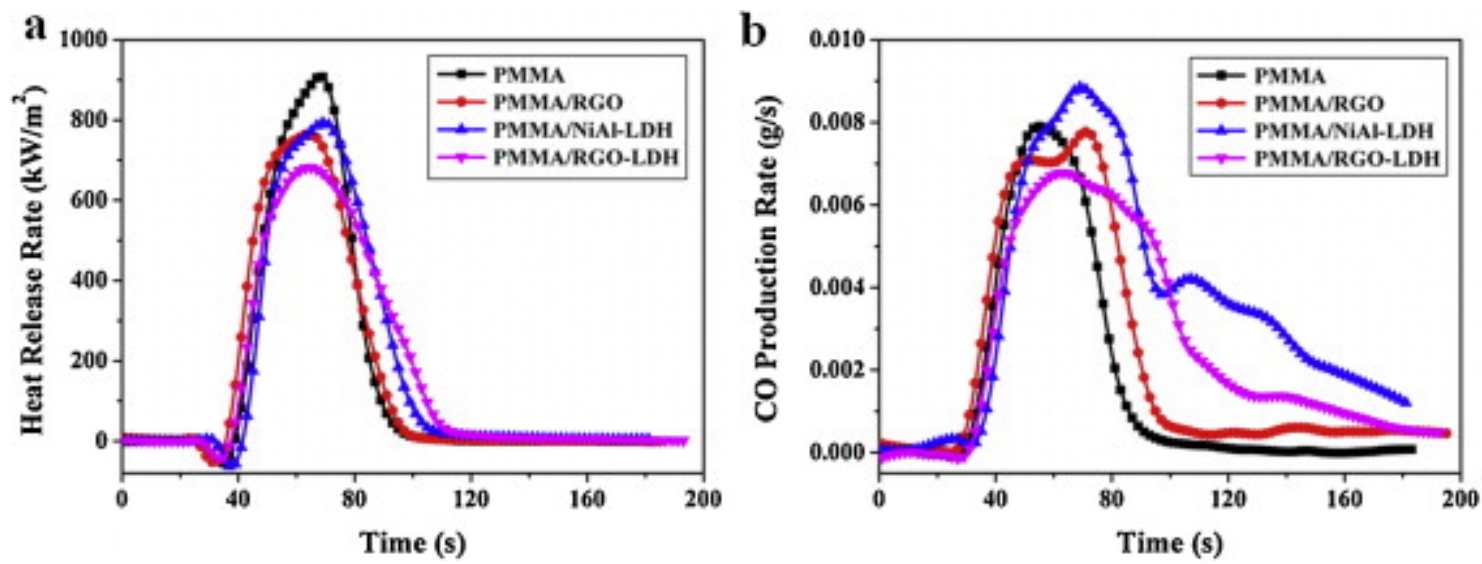

Fig. 8. Heat release rate (a) and CO production rate (b) versus time curves for pure PMMA, PMMA/RGO composite, PMMA/NiAl-LDH composite and PMMA/RGO-LDH composite.

Table 3.

Cone calorimeter data for pure PMMA, PMMA/RGO composite, PMMA/NiAI-LDH composite and PMMA/RGO-LDH composite in air at $35 \mathrm{~kW} / \mathrm{m}^{2}$.

\begin{tabular}{|l|l|l|l|l|}
\hline Sample & TTI $(\mathrm{s})$ & PHRR $\left(\mathrm{kW} / \mathrm{m}^{2}\right)$ & ASEA $\left(\mathrm{m}^{2} / \mathrm{kg}\right)$ & PCOY $(\mathrm{kg} / \mathrm{kg})$ \\
\hline PMMA & 31 & 918 & 112.7 & 2.699 \\
\hline PMMA/RGO & 31 & 764 & 135.2 & 0.563 \\
\hline PMMA/NiAl-LDH & 36 & 797 & 188.1 & 0.682 \\
\hline PMMA/RGO-LDH & 34 & 688 & 78.8 & 0.033 \\
\hline
\end{tabular}

Pure PMMA burns up, but a small quantity of black residue was collected for the PMMA/RGO-LDH composite after the cone calorimeter test. The residue of PMMA/RGO-LDH composite was investigated by SEM as shown in Fig. 9a. A large amount of amorphous char and nanoparticles covered by carbon are observed in the residual char. The whole char residue seems to form a planar structure. The char residue was also characterized by XRD with the result shown in Fig. 9b. There are three characteristic peaks appearing at $44.4^{\circ}, 51.7^{\circ}$ and $76.3^{\circ}$, which can be attributed to the diffractions of (1 111 ), ( $\left.\begin{array}{llll}2 & 0 & 0\end{array}\right)$ and ( $\left.\begin{array}{lll}2 & 2 & 0\end{array}\right)$ planes of metallic Ni. It is concluded that Ni nanoparticles are formed in situ from the reduction of NiAl-LDH by the reductive pyrolytic species of PMMA [38]. The possible carbonization promoting process of PMMA by the RGO-LDH is illustrated in Fig. 9c. Firstly, the RGO-LDH hybrid is directly incorporated into PMMA to prepare the PMMA/RGO-LDH composite by solution-blending. During the combustion process, the layered RGO will prevent the pyrolytic species from escaping. Meanwhile, the supported NiAI-LDH particles are reduced in situ to Ni particles, which serve as catalytic sites for char formation. 

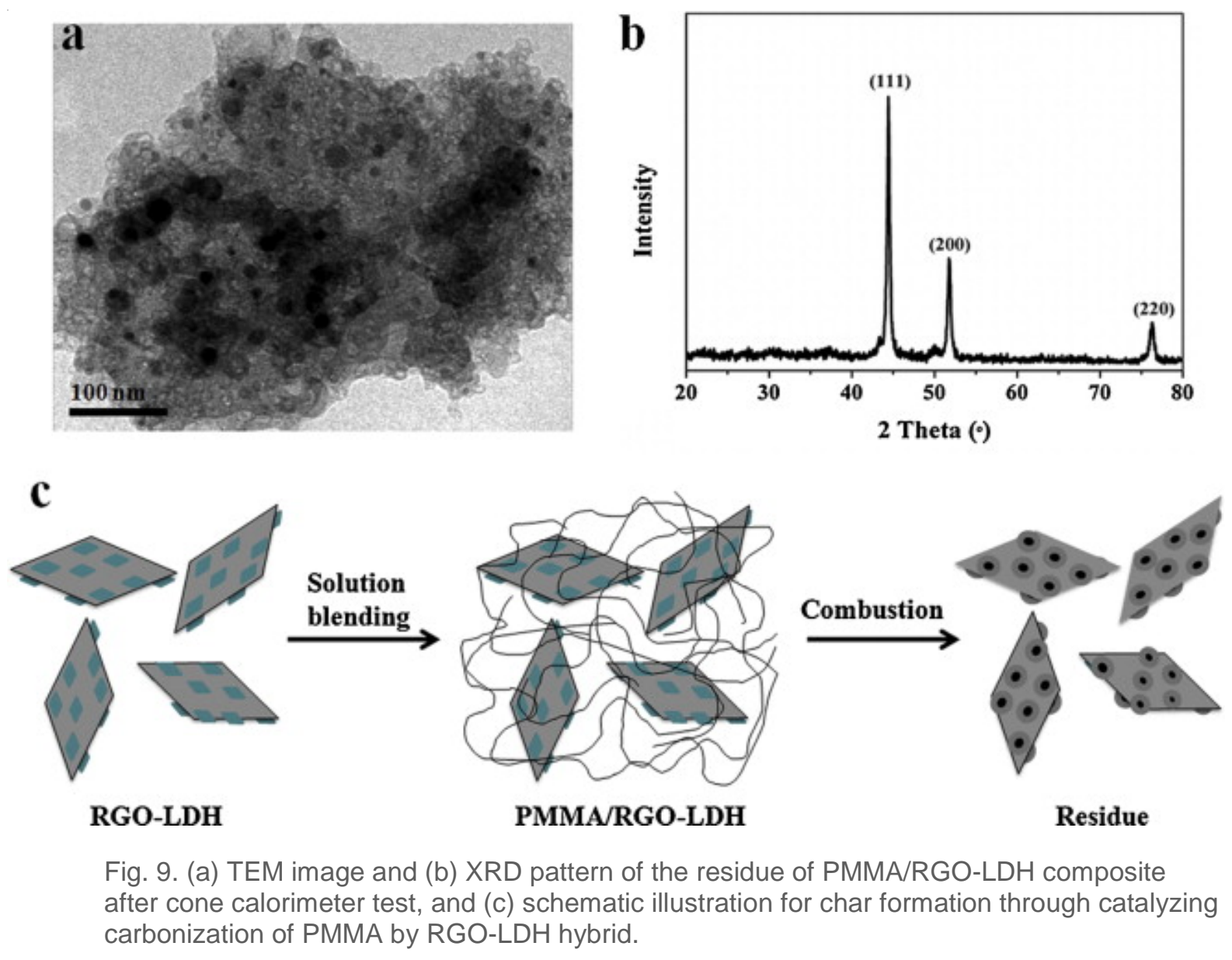

TG-IR was used to analyze differences between the pyrolytic products from PMMA and PMMA/RGO-LDH composite during thermal degradation (Fig. 10). It is observed that the maximum volatilized products of PMMA appear at 17.6 min whereas those of PMMA/RGO-LDH appear at $18.4 \mathrm{~min}$. In addition, the quantity of gaseous species from the latter is lower than that from the former. The main pyrolytic species of PMMA are hydrocarbons ( 2960 and $1448 \mathrm{~cm}^{-1}$ ), carbonylcontaining compounds $\left(1749 \mathrm{~cm}^{-1}\right)$, aromatic compounds $\left(1632 \mathrm{~cm}^{-1}\right)$, and aliphatic ethers $\left(1165 \mathrm{~cm}^{-1}\right)$ [37] and [39]. The delay of peak appearance is attributed to the barrier effect of RGO and the reduction in the quantity of volatilized products is mainly due to the catalytic role of NiAlLDH [33]. The combined effect of RGO and NiAl-LDH results in the improved flame retardancy of PMMA composite. 

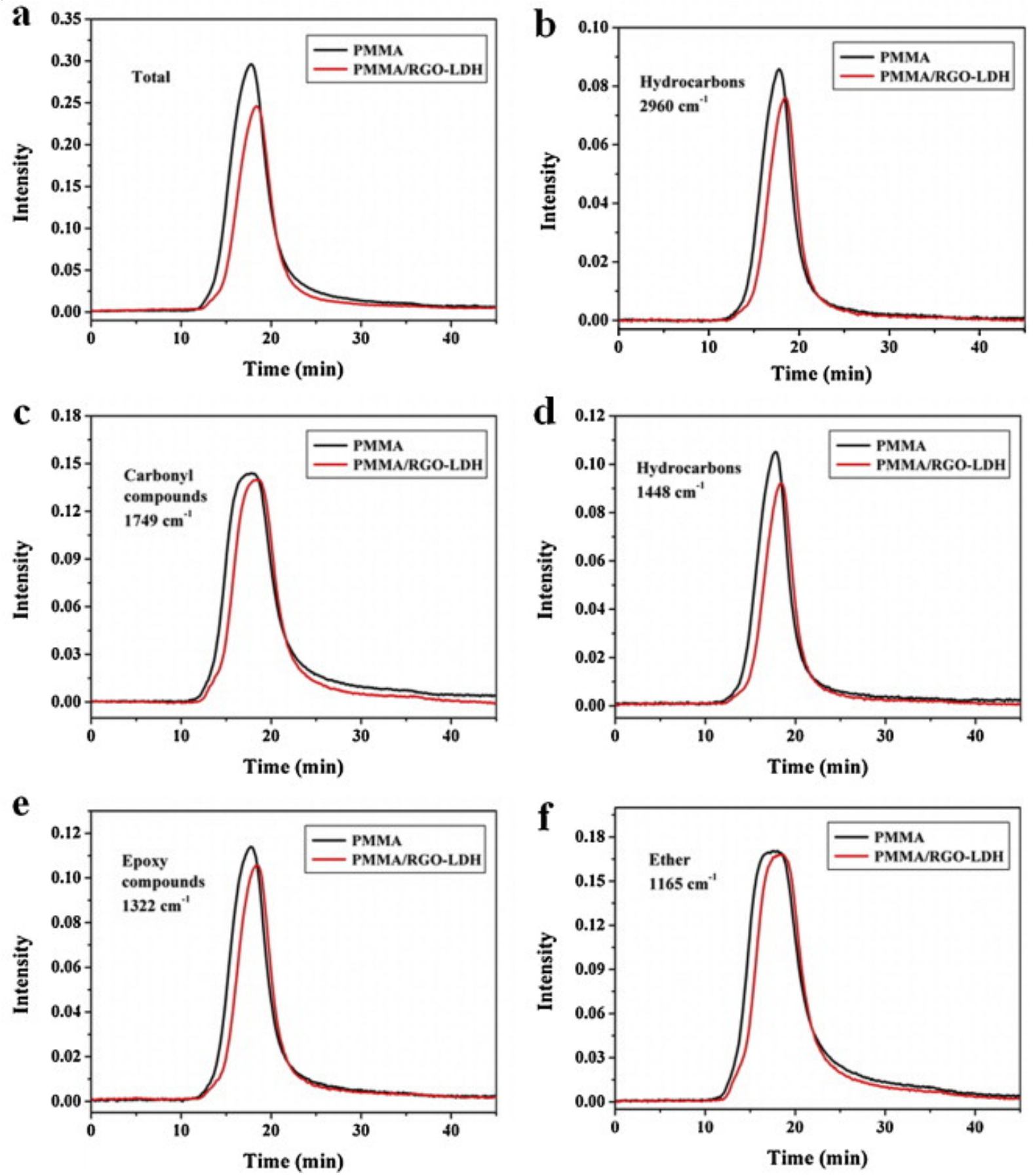

Fig. 10. FTIR absorbance of pyrolysis products for PMMA and PMMA/RGO-LDH composite versus time: (a) total, (b) hydrocarbons, (c) carbonyl compounds, (d) hydrocarbons, (e) epoxy compounds and (f) ether.

\section{Conclusions}

A hybrid RGO-LDH has been successfully synthesized by a one-pot co-precipitation method, and its structure was characterized by XRD, FTIR, Raman spectroscopy, TEM and TGA. NiAI-LDH nanoparticles with a uniform diameter of 6-10 nm were homogeneously anchored on the RGO nanosheets, which were simultaneously reduced from $\mathrm{GO}$. In both nitrogen and air, the thermal decomposition of RGO-LDH contained three steps and a high quantity of residue was left. RGO-LDH was well dispersed in PMMA and its influence on reducing flammability of the composite was investigated. The thermal stability of PMMA was improved with the addition of RGO-LDH, as revealed by the enhanced residual weight. Furthermore, the flame retardancy of the PMMA 
composite was also improved, as evidenced by the obvious reduction in PHRR and COPR values. The amount of residue was enhanced through promoting char formation of PMMA catalyzed by RGO$\mathrm{LDH}$, as demonstrated by the visible reduction and delay of volatile products. Such improvement was attributed to the combined effect of the physical barrier of RGO and the catalytic carbonization of NiAl-LDH.

\section{Acknowledgements}

The work was financially supported by the National Basic Research Program of China (973 Program) (2012CB719701), the joint fund of NSFC and CAAC (No. 61079015) and the Application of NanoScience of Fire Safety.

References

[1] V. Singh, D. Joung, L. Zhai, S. Das, S.I. Khondaker, S. Seal, Prog. Mater. Sci., 56 (2011), pp. 11781271

[2] G.B. Huang, J.R. Gao, X. Wang, H.D. Liang, C.H. Ge, Mater. Lett., 66 (2012), pp. 187-189

[3] A. Dasari, Z.Z. Yu, Y.W. Mai, G.P. Cai, H.H. Song, Polymer, 50 (2009), pp. 1577-1587

[4] G.B. Huang, H.D. Liang, Y. Wang, X. Wang, J.R. Gao, Z.D. Fei, Mater. Chem. Phys., 132 (2012), pp. $520-528$

[5] N. Wu, X.L. She, D.J. Yang, X.F. Wu, F.B. Su, Y.F. Chen, J. Mater. Chem., 22 (2012), pp. $17254-$ 17261

[6] M.C. Hsiao, S.H. Liao, Y.F. Lin, C.A. Wang, N.W. Pu, H.M. Tsaia, C.C.M. Ma, Nanoscale, 3 (2011), pp. 1516-1522

[7] X. Wang, L. Song, H.Y. Yang, W.Y. Xing, B. Kandola, Y. Hu, J. Mater. Chem., 22 (2012), pp. 2203722043

[8] C. Chen, W.T. Zhai, D.D. Lu, H.B. Zhang, W.G. Zheng, Mater. Res. Bull., 46 (2011), pp. 583-587

[9] A.S. Patole, S.P. Patole, H. Kang, J.B. Yoo, T.H. Kim, J.H. Ahn, J. Colloid Interface Sci., 350 (2010), pp. $530-537$

[10] Z. Gao, J. Wang, Z.S. Li, W.L. Yang, B. Wang, M.J. Hou, Y. He, Q. Liu, T. Mann, P.P. Yang, M.L. Zhang, L.H. Liu, Chem. Mater., 23 (2011), pp. 3509-3516

[11] H.F. Zhu, P.G. Tang, Y.J. Feng, L.J. Wang, D.Q. Li, Mater. Res. Bull., 47 (2012), pp. 532-536

[12] X.Y. Dong, L. Wang, D. Wang, C. Li, J. Jin, Langmuir, 28 (2012), pp. 293-298

[13] D. Chen, X.Y. Wang, T.X. Liu, X.D. Wang, J. Li, ACS Appl. Mater. Inter., 2 (2010), pp. 2005-2011

[14] C. Manzi-Nshuti, D.Y. Wang, J.M. Hossenlopp, C.A. Wilkie, Polym. Degrad. Stabil., 94 (2009), pp. 705-711

[15] B.X. Du, Z.P. Fang, Nanotechnology, 21 (2010), p. 315603

[16] L.J. Zhang, X.G. Zhang, L.F. Shen, B. Gao, L. Hao, X.J. Lu, F. Zhang, B. Ding, C.Z. Yuan, J. Power Sources, 199 (2012), pp. 395-401

[17] X.L. Wu, L. Wang, C.L. Chen, A.W. Xu, X.K. Wang, J. Mater. Chem., 21 (2011), pp. 17353-17359 
[18] M.X. Li, J.E. Zhu, L.L. Zhang, X. Chen, H.M. Zhang, F.Z. Zhang, S.L. Xu, D.G. Evans, Nanoscale, 3 (2011), pp. 4240-4246

[19] X.P. Zeng, J.J. Yang, W.X. Yuan, Eur. Polym. J., 48 (2012), pp. 1674-1682

[20] U. Khan, P. May, A. O’Neill, J.N. Coleman, Carbon, 48 (2010), pp. 4035-4041

[21] F.C. Chiu, I.N. Huang, Polym. Test., 31 (2012), pp. 953-962

[22] S. Park, J.H. An, R.D. Piner, I. Jung, D.X. Yang, A. Velamakanni, S.T. Nguyen, R.S. Ruoff, Chem. Mater., 20 (2008), pp. 6592-6594

[23] A.B. Bourlinos, D. Gournis, D. Petridis, T. Szabo, A. Szeri, I. Dekany, Langmuir, 19 (2003), pp. 6050-6055

[24] Q.Y. Liu, G.L. Fan, S.Y. Zhang, Y.C. Liu, F. Li, Mater. Lett., 82 (2012), pp. 4-6

[25] V. Rives, S. Kannan, J. Mater. Chem., 10 (2000), pp. 489-495

[26] J.F. Shen, M. Shi, H.W. Ma, B. Yan, N. Li, M.G. Ye, Mater. Res. Bull., 11 (2011), pp. 2077-2083

[27] J. Perez-Ramirez, G. Mul, J.A. Moulijn, Vib. Spectrosc., 27 (2001), pp. 75-88

[28] Z.P. Liu, R.Z. Ma, M. Osada, N. Iyi, Y. Ebina, K. Takada, T. Sasaki, J. Am. Chem. Soc., 128 (2006), pp. $4872-4880$

[29] H. Wang, X. Xiang, F. Li, J. Mater. Chem., 20 (2010), pp. 3944-3952

[30] C.H. Li, K.F. Yao, J. Liang, Appl. Catal. A: Gen., 261 (2004), pp. 221-224

[31] S. Morimune, T. Nishino, T. Goto, ACS Appl. Mater. Inter., 4 (2012), pp. 3596-3601

[32] M. Fang, Z. Zhang, J.F. Li, H.D. Zhang, H.B. Lu, Y.L. Yang, J. Mater. Chem., 20 (2010), pp. 96359643

[33] C. Nyambo, D. Chen, S.P. Su, C.A. Wilkie, Polym. Degrad. Stabil., 94 (2009), pp. 1298-1306

[34] T. McNally, P. Potschke, P. Halley, M. Murphy, D. Martin, S.E.J. Bell, G.P. Brennan, D. Bein, P. Lemoine, J.P. Quinn, Polymer, 46 (2005), pp. 8222-8232

[35] Y.Q. Guo, C.L. Bao, L. Song, B.H. Yuan, Y. Hu, Ind. Eng. Chem. Res., 50 (2011), pp. 7772-7783

[36] C. Nyambo, P. Songtipya, E. Manias, M.M. Jimenez-Gasco, C.A. Wilkie, J. Mater. Chem., 18 (2008), pp. 4827-4838

[37] X. Wang, L. Song, H.Y. Yang, W.Y. Xing, H.D. Lu, Y. Hu, J. Mater. Chem., 22 (2012), pp. 3426-3431

[38] H.O. Yu, Z.J. Zhang, Z. Wang, Z.W. Jiang, J. Liu, L. Wang, D. Wan, T. Tang, J. Phys. Chem. C, 114 (2010), pp. 13226-13233

[39] K. Wu, Y. Hu, L. Song, H.D. Lu, Z.Z. Wang, Ind. Eng. Chem. Res., 48 (2009), pp. 3150-3157 